\title{
A fixed agreement? Consequences of brood size manipulation on alternation in blue tits
}

\author{
Maaike Griffioen $^{\text {Corresp., }}{ }^{1}$, Wendt Müller ${ }^{1}$, Arne Iserbyt $^{1}$ \\ ${ }^{1}$ Department of Biology, Behavioural Ecology and Ecophysiology Research Group, Universiteit Antwerpen, Wilrijk, Antwerpen, Belgium \\ Corresponding Author: Maaike Griffioen \\ Email address: maaike.griffioen@uantwerpen.be
}

Recent studies have proposed that conditional cooperation may resolve sexual conflict over the amount of care provided by each parent. Such conditional cooperation may allow parents to equalize their investment by alternating their provisioning visits. This alternated pattern of male and female visits, i.e. alternation, is thought to stimulate each other's investment leading to higher levels of provisioning and potential benefits for offspring development. However, experimental studies testing the role of alternation as an adaptive parental strategy to negotiate the level of investment are still absent. Therefore, we manipulated blue tit (Cyanistes caeruleus) parents by temporarily changing their brood sizes to induce changes in demand and thus visit rates. Parents were expected to visit more and alternate at higher levels when confronted with an enlarged brood and thus there will be a greater potential for sexual conflict, assuming that prey sizes were constant. In contrast, in reduced broods visit rates and alternation may become lower due to the smaller investment that is needed for reduced broods. We show that the level of alternation did not differ in response to the manipulated brood sizes, despite a directional change in visit rates for enlarged and reduced broods as expected. Nestlings did not benefit from high levels of alternation as no effects on nestling mass gain were present in either of the different manipulations. These findings indicate that alternation does not serve as a mechanism to motivate each other to feed at higher rates. Parents hence appeared to be inflexible in their level of alternation. We therefore suggest that the level of alternation might reflect a fixed agreement about the relative investment by each of the caring parents. 


\section{A fixed agreement? Consequences of brood size manipulation on}

\section{2 alternation in blue tits}

3

4

5

6

7

8

9

10

1

20

21

22

23

24

25

26

27

28

29

30

31

32

33

34

35

36

37

38
Maaike Griffioen ${ }^{1}$, Wendt Müller ${ }^{1}$, Arne Iserbyt ${ }^{1}$

${ }^{1}$ Department of Biology, Behavioural Ecology and Ecophysiology Research Group, Universiteit Antwerpen, Wilrijk, Antwerp, Belgium

Corresponding Author:

Maaike Griffioen ${ }^{1}$

Universiteitsplein 1, Wilrijk, Antwerp, B-2610, Belgium

Email address: maaike.griffioen@uantwerpen.be 


\section{ABSTRACT}

41 Recent studies have proposed that conditional cooperation may resolve sexual conflict over the

42 amount of care provided by each parent. Such conditional cooperation may allow parents to 43 equalize their investment by alternating their provisioning visits. This alternated pattern of male 44 and female visits, i.e. alternation, is thought to stimulate each other's investment leading to 45 higher levels of provisioning and potential benefits for offspring development. However, 46 experimental studies testing the role of alternation as an adaptive parental strategy to negotiate 47 the level of investment are still absent. Therefore, we manipulated blue tit (Cyanistes caeruleus) 48 parents by temporarily changing their brood sizes to induce changes in demand and thus visit rates. Parents were expected to visit more and alternate at higher levels when confronted with an enlarged brood and thus there will be a greater potential for sexual conflict, assuming that prey sizes were constant. In contrast, in reduced broods visit rates and alternation may become lower due to the smaller investment that is needed for reduced broods. We show that the level of alternation did not differ in response to the manipulated brood sizes, despite a directional change in visit rates for enlarged and reduced broods as expected. Nestlings did not benefit from high levels of alternation as no effects on nestling mass gain were present in either of the different manipulations. These findings indicate that alternation does not serve as a mechanism to

57 motivate each other to feed at higher rates. Parents hence appeared to be inflexible in their level of alternation. We therefore suggest that the level of alternation might reflect a fixed agreement about the relative investment by each of the caring parents.

\section{INTRODUCTION}

62 Biparental care is relatively rare among species in the animal kingdom, except among bird

63 species, with more than 81 percent of the species showing this kind of care (Cockburn, 2006). To 
64 provide biparental care, two unrelated individuals get together in order to successfully raise their

65 offspring. It secures a joined fitness benefit via improved offspring development and survival,

66 however, both parents have to individually pay for the costs of providing care (Trivers, 1972).

67 Therefore, a conflict is expected between parents about the amount of investment that each

68 should make to their current brood. It is in both parents' interest that their partner provides more

69 care during the breeding attempt so that they themselves can retain energy for self-maintenance

70 and future reproduction (Stearns, 1989). How parents resolve this conflict and how this inflicts

71 their offspring has been given much attention since the formulation of the sexual conflict theory

72 by Trivers (1972). One of the first models hypothesized that each parent could only make a

73 single independent investment so that individual investment could only change on an

74 evolutionary time scale (Houston \& Davies, 1985). Whereas more recent models implemented

75 the possibility that parents are able to adjust their investment in response to their partner on a

76 behavioural time scale (Mcnamara, Gasson \& Houston, 1999; Johnstone \& Hinde, 2006; Lessells

$77 \&$ McNamara, 2012), which better captures the natural situation. However, both models predict

78 that parents should invest at suboptimal levels of care, which may reduce offspring fitness but

79 avoids exploitation by the partner (Mcnamara, Gasson \& Houston, 1999; McNamara et al., 2003;

80 Johnstone \& Hinde, 2006; Lessells \& McNamara, 2012; Johnstone et al., 2014). Recently, a

81 more cooperative strategy has been proposed for the resolution of sexual conflict, namely

82 conditional cooperation, which may avoid or reduce such costs of negotiation (Johnstone et al., 83 2014).

84

85 In humans, conditional cooperation implies that individuals are more likely to contribute to a

86 public good when others are also willing to do so, which may lead to more efficient, higher 
87 overall levels of investment (Gächter, 2007; Johnstone et al., 2014). In the context of biparental 88 care, conditional cooperation could be defined as the tendency of a parent to invest more when

89 the partner invests as well. In other words, parents may become more motivated to feed after

90 their partner has fed and therefore will speed up their visit rate. This may result in the pattern of

91 alternated feeding visits, as well as greater and more equal levels of investment (Johnstone et al.,

92 2014). Some recent studies indeed provided empirical support showing that parents alternated

93 their visits more than predicted by chance (great tits Parus major: Johnstone et al. 2014; long-

94 tailed tits Aegithalos caudatus: Bebbington \& Hatchwell, 2016; canaries Serinus canaria: Iserbyt

95 et al., 2017; chestnut-crowned babblers Pomatostomus ruficeps: Savage et al., 2017) and that

96 higher total visit rates relate to higher levels of alternation (Bebbington \& Hatchwell, 2016).

97 Consequently, this raises the question whether higher levels of alternation also benefit offspring

98 development. Evidence is still limited and confined to unmanipulated conditions, while further

99 experimental studies are needed to answer the questions whether alternation forms a parental 100 strategy to negotiate about investment and how this affects the offspring (e.g. Iserbyt et al., 101 2019).

102

103 The aim of the study was therefore to test whether the pattern of alternated male and female 104 provisioning visits is maintained when the nest conditions change, and whether higher levels of 105 alternation benefit the offspring. To this end, we performed a temporary brood size manipulation 106 experiment in blue tit (Cyanistes caeruleus) parents in which brood size was either increased or 107 decreased (Parejo \& Danchin, 2006; García-Navas \& Sanz, 2010). Previous studies have shown 108 that brood size manipulations affect parental visit rates (see review in Gow and Wiebe 2014).

109 When brood size is increased, parents are expected to increase their provisioning rate to meet the 
110 elevated brood demand, resulting in higher investment costs to both parents. Such elevated

111 demand may therefore intensify the sexual conflict, which may be ameliorated via conditional

112 cooperation through increasing individual costs of parents. When alternation improves between

113 pair members, the likelihood of exploitation may be reduced because parents motivate each other

114 to visit at higher rates. Thus alternation and visit rates are expected to rise in the situation that

115 brood size is increased, assuming that prey size remains constant. When brood size is decreased,

116 parents will likely reduce their alternation level and visit rate because of the lower sexual conflict

117 over parental care. Finally, when alternation represents a form of conditional cooperation, in

118 which parents enhance each other's willingness to invest and increase their visit rates

119 accordingly, then it should benefit the offspring. However, the offspring can only benefit from a

120 high alternation when the profitability of the delivered prey items remain unaltered. If parents

121 cheat in delivered prey sizes, then parents cannot rely on their partners' visit rate as an honest

122 signal of parental investment.

123

124

125

\section{MATERIALS \& METHODS}

Study species and measurements

126 The experiment was conducted in a nest-box population of blue tits near Antwerp, Belgium

127 (Peerdsbos $51^{0} 16^{\prime}$ N, $\left.4^{0} 29^{\prime} \mathrm{E}\right)$ from April-May 2016. Nest boxes $(\mathrm{n}=131)$ were checked twice

128 per week for nest building, egg laying and incubation. Nests were monitored daily for hatching

129 from the expected hatch date onwards. The first day of hatching was defined as day 0. Parents

130 were caught on day 6 for individual measurements and were given a plastic leg band with an

131 integrated PIT tag (which is a standard procedure in our field site and aids the individual

132 recognition by placing the PIT tag on different sides for the individuals of a pair) (Iserbyt et al.,

133 2018). Nestlings were given a unique metal ring on day 6 to allow individual recognition. 
135

136

137

138

139

140

141

142

143

144

145

146

147

148

149

150

151

152

153

154

155

156

Brood size manipulation

Each nest was subjected to a control (original brood size), reduced (minus three nestlings) and enlarged (plus three nestlings) treatment each on a separate day, i.e. post hatching day $8,9,10$ (Parejo \& Danchin, 2006; García-Navas \& Sanz, 2010). This design was chosen to control for potential confounding effects of brood age and date. The specific observation period was selected to make sure that females do not brood the young anymore (females brood nestlings 6-7 days when hatched (Perrins, 1979)), given confounding effects of task specialization on alternation (Iserbyt et al., 2017). The three nests that were chosen for the triad structure were matched for hatching date (no difference allowed), mean nestling mass (maximum difference of 1.5 gram) and brood size (maximum difference of 2 nestlings) on day 7 . When only two nests were available on the same hatch date, dyads were made considering the same selection criteria for brood size and mean nestling mass. Nests with less than 7 nestlings were not used for this experiment.

Each treatment was set up in the morning and terminated in the late afternoon (8AM-6PM). At the start of the experiment, an infrared nest-box camera (420TVL; Pakatak PAK-MIR5, Essex, UK) was placed facing downwards to the nest to record parental nest visits and prey sizes (Lucass et al., 2015). Furthermore, all nestlings were weighed individually in the morning at the start of the brood size manipulations. The three nestlings that were used for swapping between nests belonged to the intermediate positions within the weight hierarchy and did not differ more than 1 gram relative to the other selected nestlings from the other nests in the triad. Those three nestlings were taken from the nest that got the reduced treatment and were transported to the 
157 enlarged treatment nest in a cloth bag. In the late afternoon when the brood size manipulation

158 was terminated, all nestlings were weighed again and nestlings were returned to their original 159 nest.

160

161

Statistical analyses

162 Parental nest visits and prey sizes were scored from the recordings during the afternoon (starting 163 at $4 \mathrm{PM}$ ), to allow the parents to adjust to their new brood size that was manipulated in the morning. We performed a pilot analysis, in order to determine the minimum number of visits that 165 are required to provide a reliable visit rate estimate. This analysis was based on 8 control videos and showed that the average visit rate remained fairly consistent beyond 10 visits. More specifically, doubling the number of visits from 10 to 20 visits provided very similar visit rates (Pearson correlation: $\mathrm{r}=0.84 ; \mathrm{P}<0.001$ ). Therefore, the videos were analysed until each sex had at least 10 visits or the analysis was terminated after two hours when still one of the parents had not visited for 10 times. The mean time window for an analysed video recording until a parent

171 had a minimum of 10 visits, was $51.5 \pm 3.5$ minutes (mean $\pm \mathrm{SE}, \mathrm{n}=55$ ), which is likely to

172 provide accurate estimates for individual visit rates (Pagani-Núñez \& Senar, 2013; Lendvai et al., 173 2015). The size of the bird beak was used as a reference to estimate prey size: $1=$ small $(<1$

174 beak length), 2 = medium ( $1-3$ beak lengths), 3 = large ( $>3$ beak lengths) (sensu Kolliker et al., 175 1998; Lucass et al., 2015). All videos were analysed by M. Griffioen using the ObserverXT 176 program (version 10.5.572, 2011, Noldus Information Technology, The Netherlands). Males and 177 females were distinguished by their PIT tag (different sides or colour) or by their head pattern 178 (males have dark stripes on their head which are visible on the infra-red images due to the grey 179 scale of the videos). Only nests at which both parents visited were used in the analyses because 
180 we are interested in the cooperation of the parents $(n=21$ out of 23$)$. Due to failures of video

181 recordings we obtained 18 observations of control situations, 17 of reduced and 20 of enlarged.

182 Field work was carried out under the license from the Ethical Committee for animals (ECD) of

183 the University of Antwerp (license number: 2015-85).

184

185 Visit rate was calculated as visits per hour for each parent separately from the video recordings.

186 The alternation was calculated as a pair score via $\mathrm{F} /(\mathrm{t}-1)$ with $\mathrm{F}$ being the number of visits

187 that were alternated and the total number of visits (as in Bebbington \& Hatchwell, 2016). The

188 proportions of prey sizes (small, medium and large) were calculated separately per treatment for

189 each parent. Mixed effect models were used to analyse how the different parental provisioning

190 estimates varied with our brood size manipulation. The first model (linear mixed model with

191 Gaussian distribution) investigated whether variation in parental visit rate (log transformed)

192 could be explained by experimental treatment (control / reduced / enlarged), sex and the

193 interaction between treatment and sex. Brood age was included as a covariate. Parent ID nested

194 in nest ID was included as random effect to avoid pseudo-replication. The second model

195 (generalized mixed model with binomial distribution) analysed the effect of treatment on

196 parental alternation. Treatment was included as fixed factor and brood age as a covariate. Only

197 nest ID was included as a random factor because alternation is measured here as a couple

198 parameter. To further explore the effects of alternation we investigated whether visit rate

199 increased with a higher level of alternation (see Johnstone et al. 2014; Bebbington and Hatchwell

200 2016, but see Iserbyt et al. 2017). A linear mixed model (Gaussian distribution) was used in

201 which variation in the sum of male and female visit rates (total provisioning, log transformed)

202 was explained by alternation score, treatment and their interaction. Nest ID was added to the 
203 model as random effect. The fourth and fifth model (generalized mixed models with binomial

204 distribution) investigated the effect of the brood size manipulation on prey sizes. The fraction of

205 small and medium prey sizes were used as dependent variables in these models. Treatment, sex

206 and the interaction between treatment and sex were used as fixed factors and brood age as

207 covariate. The random factor was parent ID nested in nest ID. The large-sized prey were not

208 analysed because the proportions were too low and zero-inflated. To investigate whether and

209 how alternation affects offspring growth, a linear mixed model (Gaussian distribution) was fitted

210 with the change in nestling mass as response variable. Nestling mass was taken each day at the

211 start and the end of the brood size manipulation, resulting in a mean weight gain for each nest

212 (only resident nestlings). These weight gains were corrected for the variation in time period

213 between both measurements so we acquired an average nestling mass gain per hour for each nest.

214 The total provisiong, alternation scores, treatment and the interaction between treatment and

215 alternation were included in the model as a fixed effects and brood age as covariate. One outlier

216 in mass gain was removed from the analyses to get the model residuals normally distributed.

217 All mixed models were run using the package lme4 (Bates et al., 2015) and lmerTest

218 (Kuznetsova, Brockhoff \& Christensen, 2017) in R studio (version 1.1.423 and R version 3.4.3,

219 R core team, 2017). To investigate the significance of the fixed factors we performed backward

220 stepwise elimination with a critical $\alpha$ level of 0.05 . In two models the response variables 'visit

221 rate' and 'total provisioning' were log transformed to meet the normality of assumption of the

222 model residuals, tested with a Shapiro normality test and visual inspection of the residuals.

223

224 RESULTS

225 Parental feeding rates 
226 There was no significant interaction effect between treatment and sex on parental visit rates $\left(\mathrm{F}_{2}\right.$,

$22769.5=0.30, \mathrm{P}=0.739$; figure 1). Parents changed their visit rate according to experimentally

228 manipulated brood sizes $\left(\mathrm{F}_{2,70.3}=18.7, \mathrm{P}<0.0001\right)$. Specifically, parents decreased their visit rate

229 from control (unmanipulated) to reduced brood sizes (differences of LSmeans: $\mathrm{t}=2.73$, $\mathrm{df}=$

$23068.9, \mathrm{P}=0.008$ ), and increased from control to enlarged brood sizes (differences of LSmeans: $\mathrm{t}=$

$231-3.22, \mathrm{df}=67.5, \mathrm{P}=0.002$ ). Furthermore, females had an overall lower visit rate than males

$232\left(\mathrm{~F}_{1,20.4}=9.75, \mathrm{P}=0.005\right.$; figure 1$)$. Brood age also had a significant effect $\left(\mathrm{F}_{2,70.2}=5.72, \mathrm{P}=\right.$

233 0.005). The visit rate was higher when nestlings were 8 days old when compared to 9 days old

234 nestlings (differences of LSmeans: $\mathrm{t}=3.34, \mathrm{df}=69.5, \mathrm{P}=0.0014$ ) but not with 10 day old

235 nestlings (differences of LSmeans: $\mathrm{t}=1.60, \mathrm{df}=69.0, \mathrm{P}=0.113$ ).

236

237

\section{Parental alternation}

238 The alternation of feeding visits did not change as a function of our brood size manipulation $\left(\chi^{2}\right.$

$239=1.15, \mathrm{df}=2, \mathrm{P}=0.564$; see figure 2$)$. Brood age also did not significantly affect alternation $\left(\chi^{2}\right.$

$240=4.68, \mathrm{df}=2, \mathrm{P}=0.096)$. The analyses of the model with total parental visit rates revealed no

241 interaction effect of treatment with alternation $\left(\mathrm{F}_{2,38.7}=0.192, \mathrm{P}=0.826\right.$; see figure 3$)$ and as

242 well no overall relationship with alternation scores $\left(\mathrm{F}_{1,48.7}=0.027, \mathrm{P}=0.870\right)$ or brood age

$243\left(\mathrm{~F}_{2,34.1}=3.07, \mathrm{P}=0.059\right)$.

244

245 Prey sizes

246 The effect of the brood size manipulation on the proportion of the small prey size did not differ

247 between the sexes $\left(\chi^{2}=3.65, \mathrm{df}=2, \mathrm{P}=0.161\right.$; see figure 4$)$. However, the brood size

248 manipulation had a significant effect (treatment $\chi^{2}=8.54, \mathrm{df}=2, \mathrm{P}=0.014$ ) with birds bringing 
249 a higher proportion of small prey items in the control treatment when compared to the enlarged

250 treatment (post hoc Tukey: enlarged - control $\mathrm{Z}=-2.91, \mathrm{P}=0.010$ ), whilst there was no

251 difference in proportion of small prey items when control was compared to the reduced treatment

252 (post hoc Tukey: reduced - control $\mathrm{Z}=-1.47, \mathrm{P}=0.304$ ). Overall males brought more small prey

253 items than females (post hoc Tukey: $\mathrm{Z}=2.73, \mathrm{P}=0.006$ ). Brood age did not affect the

254 proportion of small prey brought to the nest $\left(\chi^{2}=4.39, \mathrm{df}=2, \mathrm{P}=0.116\right)$.

255

256 The analyses of the proportion of medium prey sizes revealed similar effects. There was no

257 significant interaction effect of treatment and $\operatorname{sex}\left(\chi^{2}=0.78, \mathrm{df}=2, \mathrm{P}=0.677\right)$. Brood size

258 manipulation hada significant effect on the proportion of medium prey (treatment: $\chi^{2}=7.43$, df $=$

$2592, \mathrm{P}=0.024)$, with the proportion of medium prey sizes being higher in the enlarged than in the

260 control brood sizes and no change between the control and reduced (post hoc Tukey: enlarged -

261 control $Z=2.64, P=0.023$; reduced - control $Z=0.74, P=0.737$ ). There was a significant sex

262 effect, females brought more medium prey items compared to males $\left(\right.$ sex: $\chi^{2}=3.97$, $\mathrm{df}=2, \mathrm{P}=$

263 0.046; see figure 4). Brood age did not affect the proportion of medium prey items $\left(\chi^{2}=1.72\right.$, df $264=2, \mathrm{P}=0.422)$.

265

266 Offspring mass gain

267 There was no significant overall effect of alternation $\left(\mathrm{F}_{1,45.3}=0.135, \mathrm{P}=0.715\right)$, treatment $\left(\mathrm{F}_{2,35.2}\right.$ $268=2.53, \mathrm{P}=0.094)$, total provisioning $\left(\mathrm{F}_{1,46.1}=0.05, \mathrm{P}=0.823\right)$ and brood age $\left(\mathrm{F}_{1,33.9}=0.0003, \mathrm{P}\right.$ $269=0.989)$ on nestling mass gain. Neither did the effect of alternation on the mass gain of nestlings 270 differ between treatments $\left(\mathrm{F}_{2,43.2}=1.66, \mathrm{P}=0.202\right.$; see figure 5). 


\section{DISCUSSION}

273 Our study shows that blue tit parents adjust their visit rates in response to experimentally

274 manipulated brood sizes. Couples nevertheless maintained similar levels of cooperation as their 275 alternation of feeding visits did not change in function of the brood size manipulations. We 276 discuss the implications of our results for the resolution of parental conflict and conditional 277 cooperation theory, as well as the functional consequences for offspring development.

278

279

280

281

282 283

284

286

287 288

289

290

291

292

293

294

\section{Effects of brood size manipulation}

We hypothesized that parents when faced with extra nestlings have to increase their visit rates to meet the enhanced offspring demand, which likely reinforces sexual conflict over parental care.

Parents may negotiate and resolve this conflict by increasing the level of alternated visits, because conditional cooperation and therewith alternation should enhance each other's willingness to invest (Johnstone et al. 2014). The blue tit parents in our study indeed increased their visit rates when confronted with three extra nestling, which is in line with observations in several other species (house wrens: Bowers et al., 2014; great tits: Hinde \& Kilner, 2007; blue tits: Nur, 1984; Parejo \& Danchin, 2006; García-Navas \& Sanz, 2010). However, we did not observe an increase of the alternation levels. Therefore, the extra investment and thus the probably reinforced conflict seems not to be mediated by alternation. Furthermore, parents additionally increased their investment when confronted with extra nestlings by increasing the number of medium-sized prey while lowering the proportion of small prey items. This contrasts previous studies that bringing smaller prey items could possibly minimize the search time for prey facilitating an increase of visit rates (Nour et al., 1998; Grieco, 2002). However, both sexes show the same pattern in prey size which makes it unlikely that parents cheat in their investment 
295 via prey sizes. Yet, females brought overall more medium-sized prey items than males

296 suggesting a sexual difference in investment. However, this difference is likely mitigated by the 297 lower visit rates of females, relative to males.

298

299 For the reduced nests we hypothesized that parents would decrease their alternation due to lower 300 301

302 303 304 305 306 307 308 309

310

311

312 313 314 315 316 317 conflict about investment caused by lower brood demand. Indeed, the blue tit parents in our study did lower their visit rates as predicted. However, again their alternation level was not changed. Furthermore, prey sizes were not altered in the reduced manipulations by the parents. When brood demand and consequently visit rate is reduced, more time becomes available as potential search time for big profitable prey (Nour et al., 1998; Grieco, 2002). However, both males and females did not elevate the small or medium prey sizes. The prey size that they brought to the nest might have been optimal in terms of foraging efficiency which is determined by the search time and energetic value of the prey (Naef-Daenzer \& Keller, 1999; Naef-Daenzer, Naef-Daenzer \& Nager, 2000).

10 A meta-analysis revealed that partial compensation is the general parental response in mate 11 handicapping or mate removal studies (Harrison et al., 2009). However, manipulation of one or 312 both parents can also result in no response (e.g. Schwagmeyer, Mock \& Parker, 2002; Santema et 13 al., 2017) and even a matching response (Hinde, 2006; Meade et al., 2011). Such variation in 14 parental responses may in part stem from multitude of applied methods and may even be observed 15 within the same study. For example in the same blue tit population which is used here, a sexspecific partial compensation was observed in response to a temporal mate removal experiment,

17 but a clear matched response followed upon the reunion of the pair (Iserbyt et al., 2019). 
318 Interestingly, we here found that male and female parents responded in similar, matched ways to

319 the brood size manipulation (García-Navas \& Sanz, 2010), which allows limited fluctuations in 320 within-pair alternation levels (see further, Bebbington \& Hatchwell, 2016).

321

322

323

324

325

326

327

328

329

330

331

332

333

334

335

336

337

338

339

340

Effects on offspring development

If alternation represents a form of conditional cooperation, in which parents enhance each other's willingness to invest, it should benefit the offspring development. Here, we did not find a relationship between total provisioning and alternation which suggests that there is no positive effect for nestlings (see also Iserbyt et al. 2017, but see Bebbington and Hatchwell 2016). Consequently, there was no evidence that higher levels of alternation resulted in increased nestling mass gain. However, the duration of the manipulation might have been too short to detect any differences in mass gain. Unfortunately, studies investigating the effects of conditional cooperation on offspring development are still limited and thus far have shown contradicting results (benefit: increased brood success, Bebbington and Hatchwell 2016; no effect: offspring development and physiological state; Bebbington and Hatchwell 2016; Iserbyt et al. 2017). That renders it difficult to draw definite conclusions about the adaptive significance and fitness consequences of alternation. We suggest that future research should not only incorporate measures of offspring development, but also estimates of parental fitness. In particular since conditional cooperation should be costly for the caring parents, or at least for the parent with the lowest physiological condition.

\section{CONCLUSIONS}


341 Alternation of parental nest visits is thought to be the outcome of a behavioural mechanism in

342 which both parents co-adjust their visit rates, thereby avoiding exploitation by their partner

343 (Johnstone et al. 2014). However, in our study we did not find such evidence that alternation and

344 visit rates (total visit rates) were related (Iserbyt et al. 2017, but see Bebbington and Hatchwell

345 2016). Furthermore, alternation remained rather constant regardless of experimentally induced

346 changes in parental visit rates. Consequently, parents might have had a fixed agreement about the

347 level of alternation (Iserbyt et al. 2019). They might use this to control for any free riding of their

348 partner and it might have already been established during earlier phases of the reproductive

349 cycle. Thus independent of the environmental conditions and brood demand, the proportion of

350 alternated visits should be kept relatively constant within pairs. This, however, does not

351 necessarily require that all visits should be alternated, especially when it might be costly to

352 monitor each other. A specific fraction of monitoring the partner's feeding behaviour might

353 provide a sufficient estimate of its investment and could serve as a signal to avoid substantial

354 exploitation. This might imply that the level of alternation should thus mainly vary among but

355 not within couples. The variation in alternation among couples could relate to quality differences

356 between parents or the compatibility of pairs (see e.g. Ihle, Kempenaers \& Forstmeier, 2015).

357 However, this remains as yet speculative as we do not know if and when such an agreement is

358 established. In this context, further research needs to specifically consider how differences in 359 quality of parents within a pair affect the alternation levels, as they have the potential to lower

360 the levels of care towards the parent of lower quality. Furthermore, environmental factors that

361 can shape the distribution of provisioning visits via prey abundance and patchy distributions in

362 the pairs' environment should be investigated to understand the role of environmental effects on 363 alternation. 


\section{ACKNOWLEDGEMENTS}

366

We thank B. Briesen, P. Scheys and N. Fresneau for assistance in the field, J.M. Baert and J.L.

Savage for statistical advice and W.F.M. van Andel for general support and discussion. A.

368

Lendvai and an anonymous reviewer for providing constructive comments, which considerably

369

helped to improve the clarity of this manuscript.

370

371

REFERENCES

372

373

Bates D, Maechler M, Bolker B, Walker S. 2015. Fitting linear mixed-effects models using lme4. Journal of Statistical Software 67:1-48. DOI: 10.18637/jss.v067.i01.

375

376

377

378

379

380

381

382

383

384

385

386

387

388

Bebbington K, Hatchwell BJ. 2016. Coordinated parental provisioning is related to feeding rate and reproductive success in a songbird. Behavioral Ecology 27:652-659. DOI: 10.1093/beheco/arv198.

Bowers EK, Nietz D, Thompson CF, Sakaluk SK. 2014. Parental provisioning in house wrens: effects of varying brood size and consequences for offspring. Behavioral Ecology 25:14851493. DOI: 10.1093/beheco/aru153.

Cockburn A. 2006. Prevalence of different modes of parental care in birds. Proceedings of the Royal Society B - Biological Sciences 273:1375-1383. DOI: 10.1098/rspb.2005.3458.

Gächter S. 2007. Conditional cooperation: behavioral regularities from the lab and the field and their policy implications. In: Frey BS, Stutzer A, editors. Psychology and economics: a promising new cross-disciplinary field. Cambridge (MA): MIT Press. p. 19-50.

García-Navas V, Sanz JJ. 2010. Flexibility in the foraging behavior of blue tits in tesponse to short-term manipulations of brood size. Ethology 116:744-754. DOI: 10.1111/j.14390310.2010.01788.x. 
389 Gow EA, Wiebe KL. 2014. Responses by Central-Place Foragers to Manipulations of Brood 390 Size: Parent Flickers Respond to Proximate Cues but do not Increase Work Rate. Ethology 391 120:881-892. DOI: 10.1111/eth.12259.

392 393

Grieco F. 2002. Time constraint on food choice in provisioning blue tits, Parus caeruleus: the relationship between feeding rate and prey size. Animal Behaviour 63:517-526. DOI: 10.1006/anbe.2002.3073.

Harrison F, Barta Z, Sze T, CUTHILL I, SZÉKELY T. 2009. How is sexual conflict over parental care resolved? A meta-analysis. Journal of Evolutionary Biology 22:1800-1812. DOI: 10.1111/j.1420-9101.2009.01792.x.

Hinde CA. 2006. Negotiation over offspring care? - A positive response to partner-provisioning rate in great tits. Behavioral Ecology 17:6-12. DOI: 10.1093/beheco/ari092.

Hinde CA, Kilner RM. 2007. Negotiations within the family over the supply of parental care. Proceedings of the Royal Society B: Biological Sciences 274:53-60. DOI: 10.1098/rspb.2006.3692.

Houston AI, Davies NB. 1985. The evolution of cooperation and life-history in the dunnock. Behavioural Ecology:471-487.

Ihle M, Kempenaers B, Forstmeier W. 2015. Fitness Benefits of Mate Choice for Compatibility in a Socially Monogamous Species. PLoS biology 13. DOI: 10.1371/journal.pbio.1002248. Iserbyt A, Fresneau N, Kortenhoff T, Eens M, Müller W. 2017. Decreasing parental task specialization promotes conditional cooperation. Scientific Reports 7:6565. DOI: 10.1038/s41598-017-06667-1.

Iserbyt A, Griffioen M, Borremans B, Eens M, Müller W. 2018. How to quantify animal activity from radio-frequency identification ( RFID ) recordings. Ecology and Evolution 8:10166- 
413

414

Iserbyt A, Griffioen M, Eens M, Müller W. 2019. Enduring rules of care within pairs - how blue tit parents resume provisioning behaviour after experimental disturbance. Scientific Reports 9:2776. DOI: 10.1038/s41598-019-39139-9.

Johnstone RA, Hinde CA. 2006. Negotiation over offspring care - how should parents respond to each other's efforts? Behavioral Ecology 17:818-827. DOI: 10.1093/beheco/arl009.

Johnstone RA, Manica A, Fayet AL, Stoddard MC, Rodriguez-Girones MA, Hinde CA, Stoddard C, Rodriguez-gironés MA, Hinde CA. 2014. Reciprocity and conditional cooperation between great tit parents. Behavioral Ecology 25:216-222. DOI: 10.1093/beheco/art109.

Kolliker M, Richner H, Werner I, Heeb P. 1998. Begging signals and biparental care: nestling choice between parental feeding locations. Animal Behaviour 55:215-22. DOI: 10.1006/anbe.1997.0571.

Kuznetsova A, Brockhoff P, Christensen R. 2017. lmerTest Package: Tests in Linear Mixed Effects Models. Journal of Statistical Software 82:1-26. DOI: 10.18637/jss.v082.i13.

Lendvai ÁZ, Akçay Ç, Ouyang JQ, Dakin R, Domalik AD, St. John PS, Stanback M, Moore IT, Bonier F. 2015. Analysis of the optimal duration of behavioral observations based on an automated continuous monitoring system in tree swallows (Tachycineta bicolor): Is one hour good enough? PLoS ONE 10:1-11. DOI: 10.1371/journal.pone.0141194.

Lessells CM, McNamara JM. 2012. Sexual conflict over parental investment in repeated bouts: negotiation reduces overall care. Proceedings of the Royal Society B: Biological Sciences 279:1506-1514. DOI: 10.1098/rspb.2011.1690.

Lucass C, Korsten P, Eens M, Müller W. 2015. Within-family parent-offspring co-adaptation in 
a wild bird: on static traits, behavioural reaction norms, and sex differences. Functional Ecology 30:274-282. DOI: 10.1111/1365-2435.12492.

437 Mcnamara JM, Gasson CE, Houston AI. 1999. Incorporating rules for responding into

438 evolutionary games. Nature 401:368-371. DOI: 10.1038/43869.

439 McNamara JM, Houston AI, Barta Z, Osorno JL. 2003. Should young ever be better off with one 440 parent than with two? Behavioral Ecology 14:301-310. DOI: 10.1093/beheco/14.3.301.

441 Meade J, Nam K-B, Lee J-W, Hatchwell BJ. 2011. An Experimental Test of the Information 442 Model for Negotiation of Biparental Care. PLoS ONE 6:e19684. DOI:

$443 \quad$ 10.1371/journal.pone.0019684.

444 Naef-Daenzer B, Keller LF. 1999. The foraging performance of great and blue tits (Parus major 445 and P. caeruleus) in relation to caterpillar development, and its consequences for nestling 446 growth and fledging weight. Journal of Animal Ecology 68:708-718. DOI: 10.1046/j.1365$447 \quad 2656.1999 .00318 . x$.

448 Naef-Daenzer L, Naef-Daenzer B, Nager RG. 2000. Prey selection and foraging performance of 449 breeding Great Tits Parus major in relation to food availability. Journal of Avian Biology 450 31:206-214. DOI: 10.1034/j.1600-048X.2000.310212.x.

451 Nour N, Currie D, Matthysen E, Van Damme R, Dhondt AA. 1998. Effects of habitat 452 fragmentation on provisioning rates, diet and breeding success in two species of tit (great tit 453 and blue tit). Oecologia 114:522-530. DOI: 10.1007/s004420050476.

454 Nur N. 1984. The Consequences of Brood Size for Breeding Blue Tits I . Adult Survival, 455 Weight Change and the Cost of Reproduction. Journal of Animal Ecology 53:479-496.

456 Pagani-Núñez E, Senar JC. 2013. One hour of sampling is enough: Great tit Parus major parents 457 feed their nestlings consistently across time. Acta Ornithologica 48:194-200. DOI: 
459 Parejo D, Danchin E. 2006. Brood size manipulation affects frequency of second clutches in the 460 blue tit. Behavioral Ecology and Sociobiology 60:184-194. DOI: 10.1007/s00265-005$461 \quad 0155-z$.

462 Perrins CM. 1979. British tits. London: Collins.

463 Santema P, Schlicht E, Schlicht L, Kempenaers B. 2017. Blue tits do not return faster to the nest 464 in response to either short- or long-term begging playbacks. Animal Behaviour 123:117465 127. DOI: $10.1016 /$ j.anbehav.2016.10.016.

466 Savage JL, Browning LE, Manica A, Russell AF, Johnstone RA. 2017. Turn-taking in 467 cooperative offspring provisioning: by-product of individual provisioning behaviour or 468 active response rule? Behavioral Ecology and Sociobiology 71:162. DOI: 10.1007/s00265469 017-2391-4.

470 Schwagmeyer PL, Mock DW, Parker G a. 2002. Biparental care in house sparrows: negotiation 471 or sealed bid? Behavioral Ecology 13:713-721. DOI: 10.1093/beheco/13.5.713.

472 Stearns S. 1989. Trade-offs in life-history evolution. Functional ecology 3:259-268. DOI:

$473 \quad 10.2307 / 2389364$

474 Trivers RL. 1972. Parental investment and sexual selection. In: Sexual Selection and the Descent 475 of Man 1871-1971. Chicago: Aldine, 136-207. DOI: 10.1002/ajpa.1330400226.

476 
Figure $\mathbf{1}$ (on next page)

Parental feeding rates (visits / hour, log transformed) for each treatment (reduced ( $\mathrm{n}=$ 17), control $(n=18)$, and enlarged $(n=20)$ brood size) and sex (blue triangles: males, red circles: females).

Symbols represent means $\pm \mathrm{SE}$. 


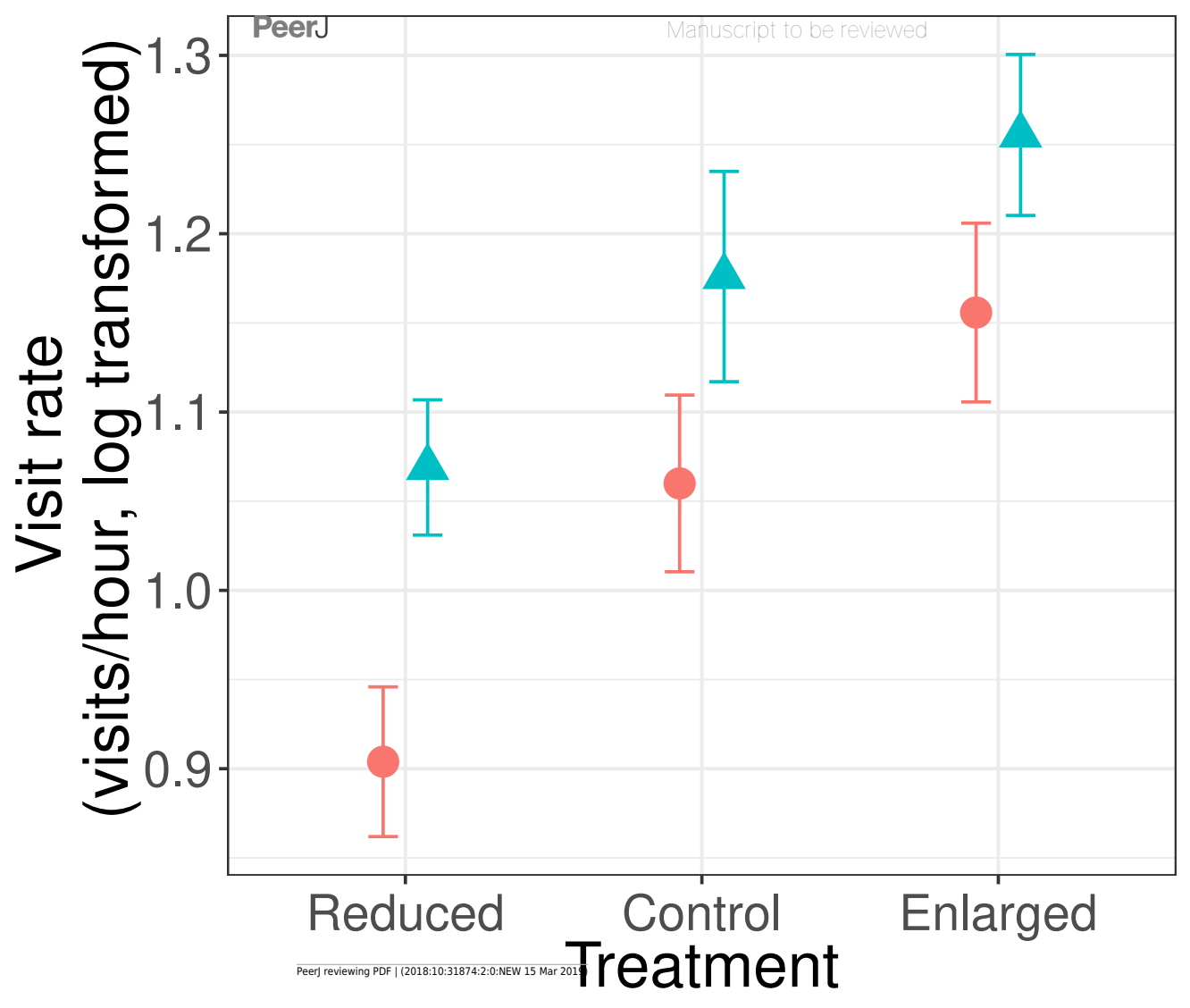




\section{Figure 2 (on next page)}

The alternation levels (number of alternated visits / total number of visits - 1) for each of the treatments (reduced $n=17$, control $n=18$, enlarged $n=20$ ).

Symbols represent means $\pm \mathrm{SE}$. 


\section{Figure 3 (on next page)}

Total parental provisioning rates (visits / hour, log transformed) per nest in relation to alternation score (number of alternated visits / total number of visits - 1).

The symbols indicate the different treatments (red circle: control brood size $(n=18)$, green triangles: enlarged $(n=20)$, and blue squares: reduced $(n=17))$. 
Figure 4 (on next page)

The proportions of prey sizes of $(A)$ males and $(B)$ females for the different treatments (reduced $(n=17)$, control $(n=18)$, and enlarged $(n=20)$ brood size).

The dark green bars (lowest bars) indicate the proportions of large prey items, medium green (middle bars) the proportions of medium-sized prey and the light green (top bars) the proportions of small prey. Symbols represent means $\pm \mathrm{SE}$. 
A) Males $\quad$ PeerJ

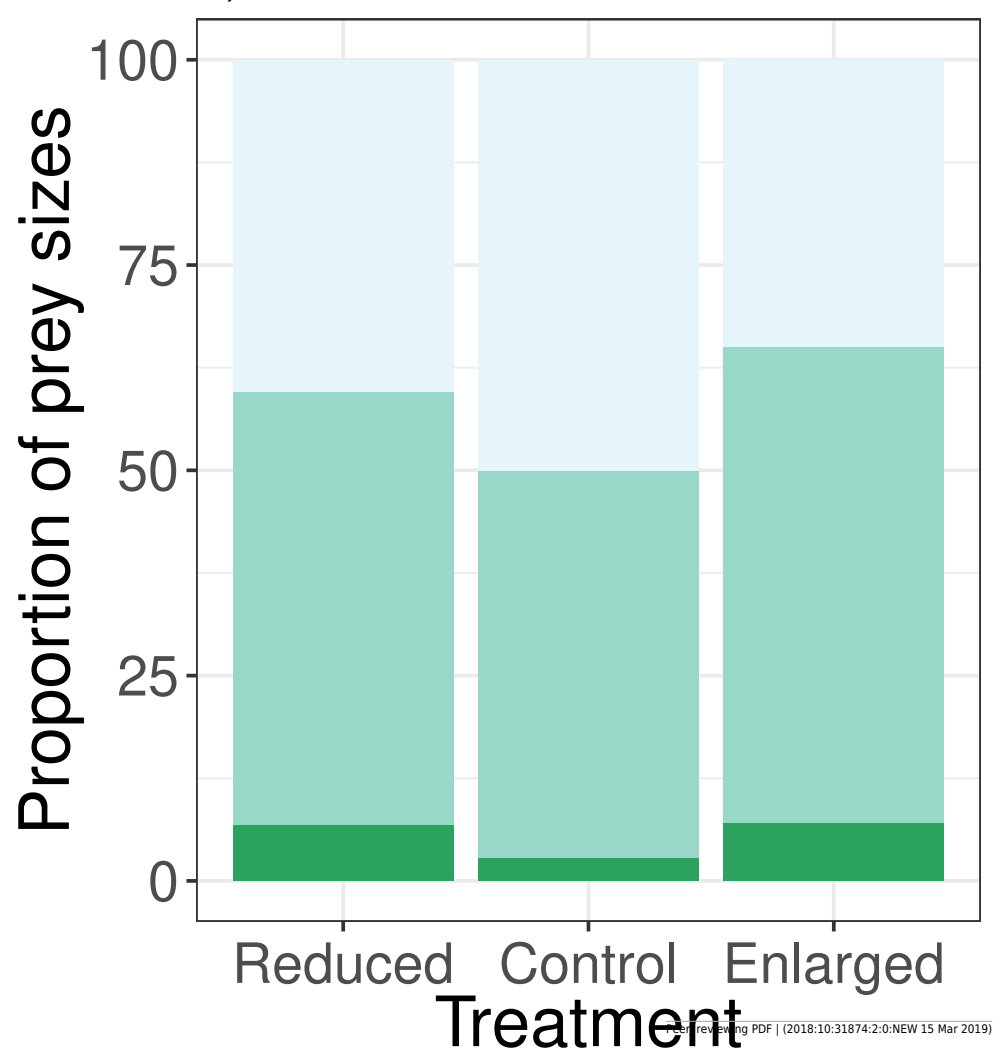

Manuscrip $\mathbf{B}$ ) Fermales

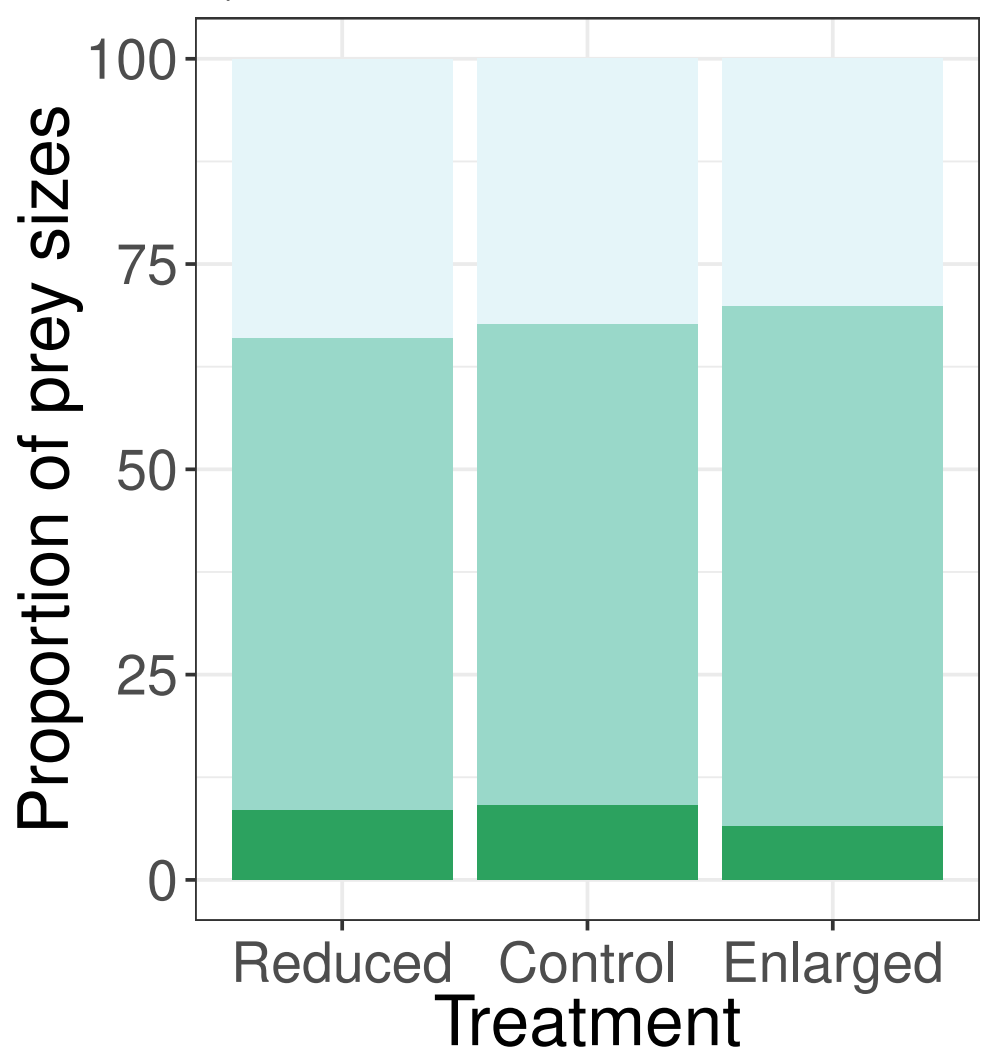


Figure $\mathbf{5}$ (on next page)

Mass gain of the nestlings (gram per hour) and alternation level of their parents.

Alternation was calculated as number of visits alternated divided by total number of visits minus one. The symbols represent the reduced (blue squares; $n=16$ (one outlier removed, see statistical analyses)), control (red circles; $\mathrm{n}=18$ ) and enlarged (green triangles; $\mathrm{n}=20$ ) treatment. 
